\title{
Evaluation of Time Consumption for Debonding Brackets Using Different Techniques: A Hospital-Based Study
}

\author{
Neelutpal Bora, ${ }^{1}$ Putul Mahanta ${ }^{1},{ }^{2}$ Ranjumoni Konwar, ${ }^{3}$ Bharati Basumatari, ${ }^{3}$ \\ Chiranjita Phukan, ${ }^{4}$ Deepjyoti Kalita $\mathbb{D}^{5},{ }^{5}$ Senjam Gojendra Singh, ${ }^{6}$ and Sangeeta Deka ${ }^{5}$ \\ ${ }^{1}$ Dentistry, Assam Medical College and Hospital, Dibrugarh 786002, Assam, India \\ ${ }^{2}$ Forensic Medicine and Toxicology, Assam Medical College and Hospital, Dibrugarh 786002, Assam, India \\ ${ }^{3}$ Radiology, Fakhruddin Ali Ahmed Medical College and Hospital (FAAMC), Barpeta, Assam, India \\ ${ }^{4}$ Medicine, Tezpur Medical College and Hospital, Tezpur, Assam, India \\ ${ }^{5}$ Microbiology, All India Institute of Medical Sciences, Rishikesh, Uttarakhand, India \\ ${ }^{6}$ Department of Psychiatry, Regional Institute of Medical Sciences, Imphal, India \\ Correspondence should be addressed to Putul Mahanta; drpmahanta@gmail.com
}

Received 21 January 2021; Revised 26 July 2021; Accepted 17 August 2021; Published 24 August 2021

Academic Editor: Antonio Gloria

Copyright ( $\odot 2021$ Neelutpal Bora et al. This is an open access article distributed under the Creative Commons Attribution License, which permits unrestricted use, distribution, and reproduction in any medium, provided the original work is properly cited.

\begin{abstract}
Background and Objectives. The debonding procedures of brackets in orthodontics cause a different amount of time loss and enamel damage. The current research assesses and equates the time consumption for bracket debonding using four different techniques. Materials and Methods. A total of 80 human premolars were included in this study. The samples were first arranged following a standard protocol for bracketing and then debonded using the ultrasonic scaler (US), debonding plier (DP), ligature cutter (LC), and thermal method (TM). Depending on the technique applied for debonding, the specimens were randomly divided into four groups with 20 samples, each keeping a 1:1 ratio. During the debonding process, the time taken for each bracket removal was recorded using a stopwatch. To assess the difference in mean time required for debonding among the four techniques, oneway ANOVA test was applied along with Tukey's HSD to compare the two methods. Results. The time range and the mean time required for the four techniques analyzed show that the DP method has the highest range of time needed for debonding with $0.97-2.56$ seconds, while LC methods have the least time range taking 0.46 to 1.79 seconds. TM's mean time to debond is the highest at 1.5880 seconds. LC method has the lowest mean debonding time of 0.9880 seconds. The one-way ANOVA test has shown the mean debonding time required by the four techniques to be significantly different $(p<0.001)$. Tukey's HSD multiple comparisons also show that the mean time to debond using the LC method is substantially less than the other three methods $(p<0.001)$. Conclusion. The mean debonding time for the TM was substantially the highest, followed by the US and DP. Debonding with the LC technique required the least time. This study shows some limelight towards the effectiveness of the LC method as it is the least time-consuming technique.
\end{abstract}

\section{Introduction}

The debonding process removes brackets with all remaining left-out adhesives from the enamel surface [1]. To restore the enamel surface, orthodontists are on the hunt for an effective and time-economic debonding method. Many have addressed the volume of enamel loss and time consumption for the bracket removal process [2-4], yet to find out the best time-economic technique of the debonding process.
The typical features of a standard orthodontic application consist of esthetical look, minimal enamel damages, and less time consumption. The dental surgeon needs to provide their patients with the appliances which have all these characters. The demand for orthodontic care has risen from $14 \%$ to $27 \%$ in recent times [5] and is thought to increase in the coming years [6].

Thus, like that of other branches, an effort for evaluating simplified and comfortable techniques of debonding from the 
patient perspective is made on [7]. Numerous orthodontists use their approach to debond the brackets on a trial basis, lacking the information of damage caused to the bracketing areas [8], including the time consumption. Hence, no agreement has reached the best bracket removal practice $[9,10]$, although the less time-consuming method was mentioned in a review [11].

Feldspar, or alumina, was merged with the first crown during the early period of the $20^{\text {th }}$ century. Leucite was then put into feldspar because of the significant discrepancies in the thermal increase of the overlying ceramic and the underlying metal alloy in the 1960s [12]. As the ceramic brackets have a notable failure rate [13], they demanded a furtherimproved method. Further ceramic bonding needs a particular etching protocol as it shows resistance to acids [14] relatively. The same research reveals the type of conditioning agent as the main factor for evaluating the bond intensity. Hence, in orthodontic practice, only the most effective method is used.

The main content of the ceramic brackets is aluminium oxide (alumina). Depending on the production procedures, the two types of ceramic brackets are used: monocrystalline and polycrystalline [15].

Aluminium oxide was first melted and then allowed to cool gradually to form the crystal to make the monocrystalline brackets. The impurities and imperfections are minimal here without the addition of the binding materials. During the process of crystallization, it is shaped like a bracket [16].

The polycrystalline brackets are produced by sintering the fragments of aluminium oxide together. The components are intermingled along with the binding material, shaped to a bracket. The shaped bracket is then fired to burn the binder, and the aluminium oxide fragments then fuse, which is an economical procedure [16].

The metal brackets have more fracture toughness in comparison to ceramic brackets. The ceramic brackets are shattered more during the time of bracket removal [17]. The process of ceramic bracket removal produces lots of inconveniences even though exceptional aesthetically. It may be associated with tie wing failure, enamel fracture, pain, and irritation during the process of bracket removal $[18,19]$. Thus, the time required for debonding may be varied. The fracture of the enamel at the time of bracket removal creates a great concern aesthetically. Furthermore, more time is needed for bracket tie wing failure for debonding by grinding with a diamond bur [20].

Still, ceramic brackets have an excellent reputation, which were introduced in the mid-1989 [21], due to their biocompatibility, attractive gazes, and best biomechanical nature in restorative dental practices [12, 22]. Yet, the metallic brackets are still considered as the gold standard as a bonding method [23].

The ceramic brackets transmit the forces to the enamel surface due to their poor flexibility at the debonding process. Therefore, appropriate care must be taken during the debonding brackets $[24,25]$, requiring different times for different techniques. Sometimes, retaining resin over the dental surface after the debonding processes creates concern as it causes enamel stain in time [26].
The enamel damage and time consumption in the debonding process depend on how the enamel surface was prepared. The class II mesio-occlusal-distal direct resin is more prone to enamel damages. However, lithium disilicate enamel rebuilding gives a good result [27].

The process of bracket removal with inappropriate methods may be durable, damaging, and aching [28]. Hence, research is required to evaluate how different debonding techniques of ceramic brackets require various times.

The current knowledge base for assessing the timeeconomic debonding technique is still inadequate. Although representing an essential contribution to our knowledge of an effective method for bracket removal, the scientific studies performed do not resolve the problem we face in selecting the patients' best preferred time-economic way of debonding.

Hence, the current research sought to weigh the time required to remove each bracket to propose an optimal debonding technique among the four methods used in the present study.

\section{Materials and Methods}

The current study included 80 left-out premolars of the patient for the orthodontic reason visiting the orthodontic department of Coorg Institute of Dental Sciences under the Rajiv Gandhi University of Health Sciences, Karnataka, Bangalore. Healthy samples without caries, fractures, or broken and structural malformations were considered and preserved in formalin solution. The enamel surface was prepared by polishing the surface with pumice, a siliceous material with a polishing brush. The brackets were debonded using four debonding techniques, i.e., US, DP, LC, and TM. The specimens were divided randomly into four groups with 20 samples, each keeping a 1:1 ratio. During debonding, the time taken to debond each bracket was recorded using a stopwatch (ACCUSPLIT Pro Survivor-A601X Stopwatch, clock, extralarge display), and the recorded time was noted for 20 specimens under each group. Afterwards, the data were statistically analyzed.

2.1. Statistical Analysis. Descriptive statistics were computed and presented as mean, standard deviation, minimum, and maximum to study the distribution of time required for debonding using each of the four techniques. To compare the difference in mean time needed for the four methods, oneway ANOVA test was applied along with Tukey's HSD for multiple comparisons. Detailed statistical analysis was done using Statistical Package for the Social Sciences (SPSS) software version 22 (IBM Corp., Armonk, New York). A $p$ value of less than 0.05 was considered statistically significant. The prior ethical clearance was obtained from the institutional ethics committee of humans (ref no. CIDS/EC/1315).

\section{Results}

The times taken to debond using the four different methods are represented in Figure 1. The time required to debond using the US method ranged from 0.86 to 2.20 seconds, while 


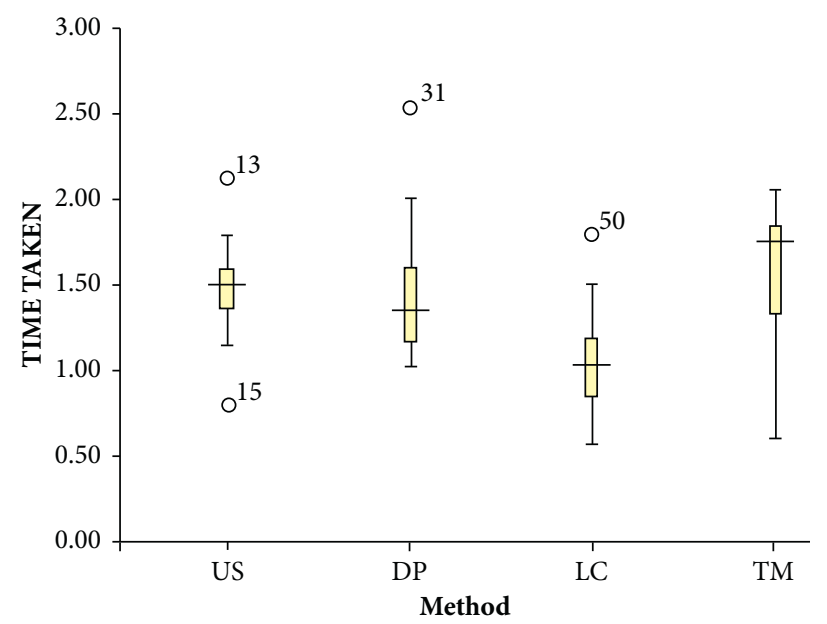

FIGURE 1: Time taken to debond by the four different methods. US: ultrasonic scaler; DP: debonding plier; LC: ligature cutter; TN: thermal method.

TABLE 1: Mean time to debond using different debonding methods.

\begin{tabular}{lcccccc}
\hline Method used & Mean & $N$ & Std. deviation & Minimum & Maximum & $F$ for one-way ANOVA $(p$ value $)$ \\
\hline US & 1.4605 & 20 & 0.27884 & 0.86 & 2.20 & $10.94(0.001)$ \\
DP & 1.4260 & 20 & 0.39531 & 0.97 & 2.56 & 1.79 \\
LC & 0.9880 & 20 & 0.31938 & 0.46 & 2.10 & \\
TM & 1.5880 & 20 & 0.40339 & 0.50 & \\
\hline
\end{tabular}

US: ultrasonic scaler; DP: debonding plier; LC: ligature cutter; TN: thermal method.

DP ranged from 0.97 to 2.56 seconds. In the TM, the required debonding time ranged from 0.50 to 2.10 seconds. The least debonding time range was 0.46 to 1.79 seconds required by the $\mathrm{LC}$ method.

The mean ( \pm standard deviation) debonding time for the TM was $1.5880( \pm 0.40339)$ seconds and was found to be significantly greater among the four debonding techniques used in this study. Debonding with the LC method required the least time with a mean ( \pm standard deviation) value of $0.9880( \pm 0.31938)$ seconds. The one-way ANOVA test revealed that the mean times to debond by the four techniques were significantly different $(p<0.001)$, as displayed in Table 1.

Tukey's HSD multiple comparisons revealed that the mean time to debond using the ligature cutter is significantly less than the other three methods $(p<0.001)$.

Simultaneously, there was no significant difference in the mean time to debond between the other three methods compared with one another, as shown in Table 2.

\section{Discussion}

The current study compared different debonding ceramic bracket methods to determine a time-efficient bracket removal technique that agrees with research [2] already done. Though studies are scanty for time evaluation following a debonding approach on ceramic brackets, several studies on the comparison of bracket removal time are available on stainless steel brackets [29-31].
As ceramic brackets' introduction to the orthodontic speciality increases its demand for its esthetic properties $[28,32,33]$, this study compares four different ceramic bracket removal methods to find the time-effective plan mentioned in some research studies [11, 20, 34]. The same tasks compared DP, US, and a mixture of both techniques. However, they reported the DP as a more time-consuming technique followed by the US, which contradicts our findings.

The mean time score for the TM of debonding was the highest, followed by the current study's US method, partially supporting a few research outcomes [20,34].

The mean time of debonding for the TM was significantly greater among the four debonding techniques. In contrast, a review reported the US as a more time-consuming method [35]. Some studies [20, 36, 37] revealed that the debonding time was minimal with the DP method than the US and electrothermal methods, partially agreeing with the current findings. However, the LC method consumed the least time significantly compared to the US, DP, and TM of debonding in the present study. In contrast, studies $[20,36,37]$ revealed insignificant differences in the debonding time between the electrothermal and conventional bracket removal methods.

4.1. Limitation of the Study. The study sample in the present study was less, and it was an in vitro study. Evaluating the relationship of debonding time with the pain induced could have been more interesting, which was not done in this study. 
TABLE 2: Tukey's multiple comparison test for the time taken between the four different debonding methods.

\begin{tabular}{|c|c|c|c|c|c|}
\hline \multirow{2}{*}{ Method (I) } & \multirow{2}{*}{ Method (J) } & \multirow{2}{*}{ Mean difference (I-J) } & \multirow{2}{*}{$p$ value } & \multicolumn{2}{|c|}{$95 \%$ confidence interval } \\
\hline & & & & Lower bound & Upper bound \\
\hline \multirow{3}{*}{ US } & $\mathrm{DP}$ & 0.03450 & 0.990 & -0.2588 & 0.3278 \\
\hline & $\mathrm{LC}$ & $0.47250^{*}$ & 0.001 & 0.1792 & 0.7658 \\
\hline & $\mathrm{TM}$ & -0.12750 & 0.665 & -0.4208 & 0.1658 \\
\hline \multirow{3}{*}{$\mathrm{DP}$} & US & -0.03450 & 0.990 & -0.3278 & 0.2588 \\
\hline & $\mathrm{LC}$ & $0.43800^{*}$ & 0.001 & 0.1447 & 0.7313 \\
\hline & $\mathrm{TM}$ & -0.16200 & 0.472 & -0.4553 & 0.1313 \\
\hline \multirow{3}{*}{ LC } & US & $-0.47250^{*}$ & 0.001 & -0.7658 & -0.1792 \\
\hline & DP & $-0.43800^{*}$ & 0.001 & -0.7313 & -0.1447 \\
\hline & TM & $-0.60000^{*}$ & 0.001 & -0.8933 & -0.3067 \\
\hline \multirow{3}{*}{$\mathrm{TM}$} & US & 0.12750 & 0.665 & -0.1658 & 0.4208 \\
\hline & $\mathrm{DP}$ & 0.16200 & 0.472 & -0.1313 & 0.4553 \\
\hline & $\mathrm{LC}$ & $0.60000^{*}$ & 0.001 & 0.3067 & 0.8933 \\
\hline
\end{tabular}

US: ultrasonic scaler; DP: debonding plier; LC: ligature cutter; TN: thermal method. ${ }^{*}$ The mean difference is significant as the $p$ value $<0.05$.

\section{Conclusion}

The ideal less time-consuming bracket removal method is challenging to evaluate as each way responds differently.

Based on the study's outcome, the mean time to debond by the four techniques was found significantly different. The mean time for the TM was considerably greater, followed by the ultrasonic scaler and debonding pliers. Debonding with the LC method requires substantially less time than that of the other three ways. Therefore, as a standard debonding method among the ceramic brackets, the LC technique may be suggested.

\section{Data Availability}

The data used to support the findings of this study are included within the article.

\section{Conflicts of Interest}

The authors declare no conflicts of interest.

\section{Acknowledgments}

The authors acknowledge the department's staff members who have helped them complete this project by providing the department's necessary infrastructure. This research was done during the postgraduate course at the Coorg Institute of Dental Sciences, Karnataka, India.

\section{References}

[1] H.-Y. Chen, M.-Z. Su, H.-F. F. Chang, Y.-J. Chen, W.-H. Lan, and C.-P. Lin, "Effects of different debonding techniques on the debonding forces and failure modes of ceramic brackets in simulated clinical set-ups," American Journal of Orthodontics and Dentofacial Orthopedics, vol. 132, no. 5, pp. 680-686, 2007.

[2] R. Gambhir, H. Khatria, R. Mangla, and H. Garg, "Evaluation of enamel surface after orthodontic debonding and cleanup using different procedures: an in vitro study," Journal of Dental Research and Review, vol. 3, no. 3, pp. 88-93, 2016.
[3] V. K. Keith, M. C. James, and S. E. Bishara, "Orthodontic bracket removal using conventional and ultrasonic debonding techniques, enamel loss, and time requirements," AJO-DO, vol. 103, no. 3, pp. 258-266, 1993.

[4] S. E. Bishara, D. Ortho., and T. S. Truiove, "Comparisons of different debonding techniques for ceramic brackets: an in vitro study," American Journal of Orthodontics and Dentofacial Orthopedics, vol. 98, no. 2, pp. 145-153, 1990.

[5] L. K. Lyons, J. D. English, and J. C. Ontiveros, "In vitro shear testing of orthodontic bonding to lithium disilicate ceramic," Journal of Cosmetic Dentistry, vol. 35, no. 1, pp. 82-89, 2019.

[6] A. H. Alzainal, S. M. Ahmed, M. Abdulfatah, A. Ani, and A. O. Mageet, "Orthodontic bonding: review of the literature," International Journal of Dentistry, vol. 2020, Article ID 8874909, 10 pages, 2020.

[7] D. H. Retief and F. R. Denys, "Finishing of enamel surfaces after debonding of orthodontic attachments," The Angle Orthodontist, vol. 49, no. 1, pp. 1-10, 1979.

[8] Y. H. Hong and K. K. K. Lew, "Quantitative and qualitative assessment of enamel surface following five composite removal methods after bracket debonding," The European Journal of Orthodontics, vol. 17, no. 2, pp. 121-128, 1995.

[9] T. Özer, G. Başaran, and J. D. Kama, "Surface roughness of the restored enamel after orthodontic treatment," American Journal of Orthodontics and Dentofacial Orthopedics, vol. 137, no. 3, pp. 368-374, 2010.

[10] T. Eliades, C. Gioka, G. Eliades, and M. Makou, "Enamel surface roughness following debonding using two resin grinding methods," The European Journal of Orthodontics, vol. 26, no. 3, pp. 333-338, 2004.

[11] M. M. Vidor, R. P. Felix, E. M. Marchioro, and L. Hahn, "Enamel surface evaluation after bracket debonding and different resin removal methods," Dental Press Journal of Orthodontics, vol. 20, no. 2, pp. 61-67, 2015.

[12] G. W. Ho and J. P. Matinlinna, "Insights on ceramics as dental materials. Part i: ceramic material types in dentistry," Siliconindia, vol. 3, no. 3, pp. 109-115, 2011.

[13] G. K. Grewal Bach, Y. Torrealba, and M. O. Lagravère, "Orthodontic bonding to porcelain: a systematic review," The Angle Orthodontist, vol. 84, no. 3, pp. 555-560, 2013.

[14] H. Kato, H. Matsumura, T. Ide, and M. Atsuta, "Improved bonding of adhesive resin to sintered porcelain with the combination of acid etching and a two-liquid silane 
conditioner," Journal of Oral Rehabilitation, vol. 28, no. 1, pp. 102-108, 2001.

[15] M. L. Swartz, "Ceramic brackets," Journal of Clinical Orthodontics: Journal of Clinical Orthodontics, vol. 22, no. 2, pp. 82-88, 1988.

[16] S. E. Bishara and D. E. Fehr, "Ceramic brackets: something old, something new, a review," Seminars in Orthodontics, vol. 3, no. 3, pp. 178-188, 1997.

[17] G. E. Scott, "Fracture toughness and surface cracks-the key to understanding ceramic brackets," The Angle Orthodontist, vol. 58 , pp. $5-8,1988$.

[18] S. E. Bishara, A. W. Ostby, J. Laffoon, and J. J. Warren, "Enamel cracks and ceramic bracket failure during debonding in vitro," The Angle Orthodontist, vol. 78, no. 6, pp. 1078-1083, 2008.

[19] T. S. Normando, F. S. Calçada, W. J. Ursi, and D. Normando, "Patients' report of discomfort and pain during debonding of orthodontic brackets: a comparative study of two methods," World Journal of Orthodontics, vol. 11, pp. e29-34, 2010.

[20] S. E. Bishara and T. S. Trulove, "Comparisons of different debonding techniques for ceramic brackets: an in vitro study," American Journal of Orthodontics and Dentofacial Orthopedics, vol. 98, no. 3, pp. 263-273, 1990.

[21] D. Birnie, "Ceramic brackets," British Journal of Orthodontics, vol. 17, no. 1, pp. 71-75, 1990.

[22] R. Ramakrishnaiah, A. Alkheraif, D. Divakar, J. Matinlinna, and P. Vallittu, "The effect of hydrofluoric acid etching duration on the surface micromorphology, roughness, and wettability of dental ceramics," International Journal of Molecular Sciences, vol. 17, no. 6, 822 pages, 2016.

[23] M.-Z. Su, E. H.-H. Lai, J. Z.-C. Chang et al., "Effect of simulated debracketing on enamel damage," Journal of the Formosan Medical Association, vol. 111, no. 10, pp. 560-566, 2012.

[24] S. E. Bishara, D. E. Fehr, and J. R. Jakobsen, "A comparative study of the debonding strengths of different ceramic brackets, enamel conditioners, and adhesives," American Journal of Orthodontics and Dentofacial Orthopedics, vol. 104, no. 2, pp. 170-179, 1993.

[25] A. Verstrynge, G. Willems, and A. Ghesquiere, "Clinical comparison of an adhesive precoated vs. an uncoated ceramic bracket system," Orthodontics and Craniofacial Research, vol. 7, no. 1, pp. 15-20, 2004.

[26] W. Carstensen, "Clinical results after direct bonding of brackets using shorter etching times," American Journal of Orthodontics, vol. 89, no. 1, pp. 70-72, 1986.

[27] P. Ausiello, S. Ciaramella, A. Fabianelli et al., "Mechanical behavior of bulk direct composite versus block composite and lithium disilicate indirect Class II restorations by CAD-FEM modeling," Dental Materials, vol. 33, no. 6, pp. 690-701, 2017.

[28] A. K. Jena, R. Duggal, and A. K. Mehrotra, "Physical properties and clinical characteristics of ceramic brackets: a comprehensive review," Trends in Biomaterials and Artificial Organs, vol. 20, no. 2, 2007.

[29] H. Khan, A Rafi Chaudhry, F. Ahmad, and F. Warriach, "Comparison of debonding time and pain between three different debonding techniques for stainless steel brackets," Pakistan Oral \& Dental Journal, vol. 35, no. 1, pp. 79-82, 2015.

[30] D. A. Flores, L. K. Choi, J. M. Caruso, J. L. Tomlinson, G. E. Scott, and M. T. Jeiroudi, "Deformation of metal brackets: a comparative study," The Angle Orthodontist, vol. 64, no. 4, pp. 283-290, 1994.

[31] S. Arici and D. Regan, "Alternatives to ceramic brackets: the tensile bond strengths of two aesthetic brackets compared ex vivo with stainless steel foil-mesh bracket bases," British Journal of Orthodontics, vol. 24, no. 2, pp. 133-137, 1997 May.

[32] A. Karamouzos, A. E. Athanasiou, and M. A. Papadopoulos, "Clinical characteristics and properties of ceramic brackets: a comprehensive review," American Journal of Orthodontics and Dentofacial Orthopedics, vol. 112, no. 1, pp. 34-40, 1997.

[33] P. M. Campbell, "Enamel surfaces after orthodontic bracket bonding," The Angle Orthodontist, vol. 2, pp. 103-110, 1995.

[34] S. E. Bishara, M. E. Olsen, and c. L. Von Wald, "Evaluation of debonding characteristics of a new collapsible ceramic bracket," American Journal of Orthodontics and Dentofacial Orthopedics, vol. 112, no. 5, pp. 552-559, 1997.

[35] S. W. Merrill, L. J. Oesterle, and C. B. Hermesch, "Ceramic bracket bonding: a comparison of shear, tensile, and torsional bond strengths of ceramic brackets," American Journal of Orthodontics and Dentofacial Orthopedics, vol. 106, no. 3, pp. 290-297, 1994.

[36] J. M. Bordeaux, R. N. Moore, and M. D. Bagby, "Comparative evaluation of ceramic bracket base designs," American Journal of Orthodontics and Dentofacial Orthopedics, vol. 105, no. 6, pp. 552-560, 1994.

[37] S. E. Bishara and T. S. Trulove, "Comparisons of different techniques for ceramic brackets: an in vitro study. part I. background and methods," American Journal of Orthodontics and Dentofacial Orthopedics, vol. 98, 1990. 